\title{
Propuesta de Intervención para Desarrollo del Capital Psicológico
}

\author{
Fernando Toro Álvarezl
}

Recibido: 12/06/2018 Aceptado: 23/10/2019

DOI: $10.21772 /$ ripo.v38n2a02

\begin{abstract}
Resumen
La literatura ha puesto en evidencia que el capital psicológico es un constructo bien fundamentado, conceptual y metodológicamente. Su utilidad y conveniencia están en su relevancia para comprender asuntos sociales y organizacionales como desempeño, bienestar subjetivo, emprendimiento o clima organizacional. Estudios recientes han mostrado la eficacia de estrategias educativas para desarrollo del capital psicológico y de cada uno de sus cuatro componentes: esperanza, eficacia, optimismo y resiliencia. No obstante, los efectos de esas estrategias no han logrado permanecer por más de tres meses. El presente escrito contiene una nueva estrategia pensada para desarrollar en las personas estas condiciones, de modo que las mejoras obtenidas persistan por períodos más amplios. Esta mejora en la permanencia del aprendizaje se fundamenta en el desarrollo de procesos de regulación de emociones positivas y en un plan de aprendizaje distribuido en varias sesiones, que ayuda a la repetición y reforzamiento de elementos cognitivos, emocionales y conductuales adquiridos en las sesiones. Este escrito sugiere alternativas de aplicación de la estrategia y criterios para su evaluación.
\end{abstract}

Palabras clave: Capital psicológico, regulación de emociones, intervención, estrategia.

\section{Intervention Proposal for Psychological Capital Development}

\begin{abstract}
Psychological Capital is a well-supported construct on theory and methodology. Its convenience and usefulness depend on its relevance to the understanding of social and organizational issues such as performance, subjective wellbeing, entrepreneurship or organizational climate. Recent studies have evidenced the effectiveness of education strategies leaded to the psychological capital development and each one of its components: hope, efficacy, optimism and resilience. The effects of such strategies have remained for no more than three months. Current paper offers a new strategy to the improvement of psychological capital that allows to keep the gains achieved for longer periods. This improvement in learning permanence is supported on the training of positive emotions regulation and a plan of distributed learning along several sessions, intended to repeat and reinforce cognitive, emotional and behavioral improvements achieved in previous sessions. Current paper considers also alternatives to apply the strategy suggested and criteria for its assessment.
\end{abstract}

Key words: Psychological capital, emotion regulation, intervention, strategy.

1 Dr. En Psicología Industrial Organizacional. Universidad de Puerto Rico Recinto Piedras Blancas. Socio fundador de Cincel S.A.S. y de la Revista Interamericana de Psicología Ocupacional. E-mail: ftoro@cincel.com.co

Cómo citar este artículo: Toro Álvarez, F. (2019). Propuesta de Intervención para Desarrollo del Capital Psicológico. Revista Interamericana de Psicología Ocupacional, 38(2), 115-126. DOI: 10.21772/ripo.v38n2a02 


\section{Propuesta de Intervención para Desarrollo del Capital Psicológico}

El capital psicológico puede entenderse como un conjunto de estados psicológicos esperanza, eficacia, optimismo y resiliencia- que habilita a la persona para experimentar bienestar y satisfacción en su vida personal y de trabajo, alcanzar un desempeño social y de tarea positivo $\mathrm{y}$ enriquecer el ambiente en el que se encuentra y participa (Avey, Reichard, Luthans y Mhatre, 2011; Lutans, Lutans y Avey, 2013). Esta condición psicosocial tan conveniente para la persona lo es también para el equipo de trabajo y para toda la organización. En la medida en que se trata de algo intermedio entre un estado de ánimo y un rasgo de personalidad, sin ser exactamente uno u otro, admite la posibilidad de desarrollo (Luthans, Avolio, Avey y Norman, 2007). Por tanto, disponer de un plan bien conformado para su mejoramiento y desarrollo es contar con una ventaja competitiva en cualquier lugar de trabajo (Trejo, Richard, Van Driel y McDonald, 2015).

El objetivo del presente escrito es proponer una estrategia para el desarrollo del capital psicológico, que conserve los efectos del aprendizaje en el mediano y largo plazo. Se encuentran en la literatura algunas experiencias educativas breves (Luthans, Luthans y Avey, 2013; Wingerden, Bakker y Derks, 2016) con un buen nivel de eficacia, pero en ellas solo se han evidenciado sus efectos en el corto plazo -no más de tres meses-. La estrategia que aquí se propone contempla una duración ligeramente mayor de la experiencia educativa, pero busca conseguir efectos de aprendizaje de más amplia duración. Para tal propósito se extiende en el tiempo la experiencia educativa de modo que los procesos de aprendizaje puedan pasar de la memoria de trabajo, hipotalámica y de corta duración a la memoria declarativa, consolidada en la corteza cerebral y de largo plazo (Eichenbaum, 2003; Squire, 2004). Además, el aprendizaje de algunos mecanismos de regulación de emociones tendrá el mismo efecto de dotar al participante de recursos individuales y colectivos que pueden prolongar en el tiempo lo aprendido.

En la primera parte del escrito se presentan de modo breve los conceptos asociados al capital psicológico, que serán luego parte de la experiencia educativa. A continuación se expone el concepto de regulación de las emociones y varios mecanismos de regulación que se aprenderán en el proceso. Luego de considerar algunos criterios esenciales de intervención se elabora la estrategia que, se espera, promueva los efectos previamente descritos.

\section{Capital psicológico}

Este es un constructo de segundo orden que surge del análisis factorial de sus cuatro factores componentes: esperanza, autoeficacia, optimismo y resiliencia (Luthans, Avey y Patera, 2008). Cada uno de los elementos que lo integran está fundamentado teórica y empíricamente. Estudios posteriores dan soporte adecuado también al concepto general de capital psicológico (Peterson, Luthans, Avolio, Walumbwa y Zhang, 2011). Estos investigadores lo entienden como un constructo multidimensional de orden superior, basado en la teoría de la conservación de recursos (Hobfoll, 2002) la cual propone que los empleados están motivados para conservar y aumentar en el tiempo los recursos necesarios para obtener resultados exitosos de su desempeño. Se ha encontrado que el capital psicológico puede ser un predictor importante de actitudes y conductas en el trabajo tanto deseables como indeseables (Avey et al., 2011). Por tratarse más de un "cuasi estado" psicológico que de un rasgo es modificable a través de acciones educativas y procesos de intervención (Luthans, Avey, Avolio y Peterson, 2010).

Luthans, Youssef y Avolio (2007) definieron el capital psicológico como un estado de desarrollo psicológico de la persona, soportado 
por cuatro atributos: (1) confianza en contar con la disposición al esfuerzo y la habilidad necesarias para teneréxito en una tarea demandante-eficacia-. (2) Asumir que ocurrirán eventos o circunstancias que aseguran la posibilidad de éxito actual o futuro -optimismo-. (3)Perseverar en el logro de las metas $\mathrm{y}$, cuando sea necesario, redirigir el camino para asegurar el éxito -esperanza-. (4) Ante el asedio de los problemas o de la adversidad, recuperarse o resurgir -resiliencia-. Se ha encontrado en la investigación que capital psicológico, como concepto y medida integral, es mejor predictor de desempeño y satisfacción que cada uno de sus componentes aislado (Luthans et al., 2007). No obstante, es necesario precisar el significado de cada uno de los factores componentes a fin de poderlos traducir a indicadores conductuales para el diseño de experiencias que promuevan su aprendizaje y desarrollo.

Eficacia. Se refiere a la convicción de contar con las habilidades, motivación y conocimiento necesarios para enfrentar con éxito una actividad. Se desarrolla a partir del manejo competente de la tarea, del aprendizaje vicario, de la observación de modelos, de la persuasión social y de la activación psicológica y fisiológica (Luthans, Avey y Patera, 2008).

Optimismo. Se entiende como una atribución personal que asume la ocurrencia de eventos positivos en el futuro, como algo más probable que la de eventos negativos futuros, y depende de experiencias previas y de las condiciones personales de cada individuo (Luthans et al., 2008).

Esperanza. Se constituye a partir de tres componentes: disposiciónala acción, característica de personas con un locus de control interno bien desarrollado; visualización de opciones alternativas que permiten hacer elecciones $\mathrm{y}$, por último, de metas o propósitos claros (Luthans et al., 2008). Estos elementos suelen aprenderse mediante acciones intencionales enfocadas en la búsqueda de soluciones (Snyder, 1994), por lo que tiene sentido pensar en la posibilidad de educar y mejorar esta condición en las personas en el trabajo.

Resiliencia. Es habilidad para recuperarse de la adversidad o el fracaso o para tomar medidas preventivas antes de que estos ocurran (Luthans et al. 2008). Se trata de una respuesta adaptativa a eventos adversos, que surge o puede surgir de la interacción con el ambiente y tiene como efectos promover el bienestar de la persona, protegerla de riesgos y facilitar su recuperación ante eventos adversos (Russo y Stoycova, 2015).

Estos constructos han mostrado una clara correlación y capacidad de explicar porciones importantes de la varianza en otras variables significativas en ambientes de trabajo. Avey et al. (2011), en un estudio de meta-análisis sobre el impacto del capital psicológico en las actitudes, conductas y desempeño del personal, encontraron evidencias empíricas acerca de efectos positivos del capital psicológico en actitudes deseables como satisfacción con el trabajo, compromiso organizacional y bienestar psicológico; en el comportamiento de ciudadanía organizacional, visto como una conducta deseable. También evidenciaron la relación con medidas diversas del desempeño como evaluaciones objetivas, autoevaluaciones o evaluaciones por el supervisor. Se encontraron reportes de correlaciones negativas con actitudes indeseables como cinismo, intención de rotación, ansiedad y estrés y también con conductas contra-productivas en el trabajo. Como dato importante en el estudio observaron que las relaciones mencionadas tendieron a ser más altas en Estados Unidos que en algunos países asiáticos y europeos y en el sector de los servicios, comparado con el manufacturero, por lo que concluyeron que el capital psicológico es predictor importante de actitudes, conductas y desempeño de las personas en el trabajo.

En el contexto de una intervención sobre las demandas y recursos en el trabajo, Wingerden et al. (2016) constataron que éstas tuvieron un 
efecto significativo sobre el capital psicológico, el que a su vez se relacionó con mejoras significativas en job crafting, engagement y desempeño de las personas participantes. Efectos positivos similares reportaron Luthans et al. (2014) en un proceso de desarrollo del capital psicológico como una experiencia académica realizada con estudiantes universitarios de administración. Efectos como los referidos evidencian que el capital psicológico y sus distintos componentes son estados con capacidad para afectar las actitudes y conducta de las personas, son susceptibles de mejorar y desarrollarse con la educación y sus efectos positivos constituyen evidentes beneficios para las personas y para las organizaciones.

El concepto de capital psicológico y sus cuatro componentes se pueden entender como fundamentados en estructuras cognitivas interactuando con procesos emocionales que las complementan y refuerzan. El vínculo entre estructuras cognitivas y procesos emocionales ha sido ampliamente documentado (Garnefski, Teerds, Kraaij, Legerstee y fan den Kommer, 2004; Tugade y Fredrickson, 2007; Lopez, Mezlec, Extremera, Hertel, Hernández, Shultz y Salovey, 2011; Quoidbach, Berry, Hansenne y Milkolajczak, 2010; Salimpoor, Van den Bosch, Kovasevic, Mcintoch, Dagher y Zatorre, 2013; Silvers y Ochsner, 2016), al igual que el vínculo estrecho entre los afectos y las actuaciones de las personas en el trabajo (Warr, Bindl, Parker y Inceoglu, (2013). Estas y otras investigaciones coinciden en que la regulación de emociones es un proceso que puede acompañar y facilitar la adquisición y consolidación de patrones cognitivos. El aprendizaje y desarrollo del capital psicológico se fundamenta en la interacción y refuerzo entre lo racional y cognitivo y lo emocional. De este modo, trabajar en la regulación de emociones positivas complementa y refuerza los procesos cognitivos y racionales inherentes al desarrollo del capital psicológico.

\section{Regulación de Emociones Positivas}

Regulación de las emociones es el manejo que las personas hacen de sus afectos de modo que puedan evitar estados indeseables o favorecer estados placenteros o positivos. Se trata de modos de control que las personas ponen en funcionamiento por el efecto que logran en términos de bienestar, adaptación al ambiente, seguridad y protección. Gross, Richards y John (2006) entienden la regulación como un intento por controlar qué tipo de emociones experimentar en una circunstancia dada, cómo experimentarlas -positiva o negativamente- y de qué manera expresarlas. Esto ocurre, explican Tugade y Fredrickson (2007), por mecanismos como la prolongación o mantenimiento de la emoción positiva experimentada o por el incremento de emociones positivas o negativas asociadas a un evento. El principio de la consistencia hedónica (Wegener y Petty, 1994) sugiere que cuando alguien está en un estado subjetivo positivo busca actividades que le permitan mantener ese estado. Pero, por otra parte, pueden activarse emociones negativas en una situación cuya regulación se dirige no a su conservación sino a su prevención o a su control una vez experimentadas (Quoidbach et al., 2010).

Estos procesos psicológicos están soportados por actividades neurales en las que participan estructuras procesadoras y reguladoras de emociones tales como la amígdala, el núcleo acumbens, el tálamo y la corteza prefrontal. Estos núcleos se integran en redes complejas que finalmente permiten percibir situaciones, experimentar emociones positivas o negativas y producir respuestas conducentes a evitar o controlar emociones negativas o a mantener e incrementar las positivas (Ernst et al., 2005; Knutson et al., 2008; Salimpoor et al., 2013).

En la literatura se reportan varios tipos de regulación de emociones. Tugade y Fredrickson (2007) estudiaron el Saboreo - "savoring"-, un tipo de estrategia con capacidad para conservar 
estados emocionales positivos. En un análisis más detallado Quoidbach et al., (2010) propusieron dos categorías de mecanismos de regulación de emociones, el saboreo y la atenuación, cada una con estrategias específicas que tienen en común, en el primer caso, el disfrute y la prolongación de la experiencia emocional, en el segundo, la atenuación, se busca la disminución o eliminación de la experiencia emocional. En la categoría de estrategias de Saboreo incluyeron Expresión Conductual (expresión no verbal de la emoción), Estar Presente (Dirigir atención deliberada a la experiencia placentera), Capitalización (comunicar y celebrar los eventos positivos), Viaje mental positivo en el tiempo (recordar vivamente o anticipar eventos positivos). En la categoría de Estrategias de Atenuación fueron incluidas: Supresión (reprimir u ocultar la emoción positiva), Distracción (realizar actividades o pensar, a menudo preocuparse, en asuntos ajenos al evento positivo). Encontrar deficiencias (atender a aspectos negativos del evento o situación o considerar lo que hubiera podido ser mejor), Viaje mental negativo en el tiempo (recordar vivamente o anticipar consecuencias negativas). Estos estudios han señalado un repertorio de procedimientos de regulación de emociones positivas que pueden utilizarse para prolongar estados de ánimo y afectos positivos o para prevenir sus efectos emocionales.

Los estudios han examinado diferentes consecuencias de las emociones positivas. Tugade y Fredrickson (2007) identificaron diversos efectos del saboreo: bienestar, afrontamiento del estrés, resiliencia, optimismo auto-reportado, internalidad, autocontrol, satisfacción con la vida y autoestima. Además, la habilidad para regular emociones jugó un papel significativo en la calidad de la interacción social, el manejo del estrés y del conflicto (Trejo et al., 2015).

En otro estudio Yoo, Miyamoto y Ryff (2016) reportaron que un alto nivel de afecto positivo y conectividad social se asociaron con altos niveles de salud, evaluados mediante varios marcadores fisiológicos. Tsai (2001) encontró en su investigación que, como efecto de la expresión de emociones positivas de los vendedores hacia los clientes en un almacén de calzado, éstos últimos tendieron a comprar más, a volver de nuevo al almacén y a comunicar a otras personas su experiencia. Trejo et al., (2015) reportaron un vínculo significativo entre la habilidad de regulación de emociones y el optimismo, entre personal militar. No obstante lo anterior, es preciso considerar que las experiencias emocionales y su regulación ocurren en un contexto social, económico, cultural que condiciona esos procesos psicológicos (Kelly y Barsade, 2001).

En otra investigación Parke y Seo (2017) observaron relaciones entre el clima de afecto y varios indicadores de desempeño organizacional: calidaddelas relaciones, productividad, creatividad y confiabilidad del desempeño. Investigaciones como las referidas destacan la relevancia de un clima que provea legitimidad a la expresión de emociones positivas, como una condición mediadora entre las prácticas administrativas y los comportamientos afectivos de las personas en el trabajo. Estos comportamientos incluyen no solo la experiencia y expresión de emociones y estados psicológicos sino también los procesos de regulación que las conservan o las modulan.

Dadas las características y los efectos del capital psicológico y de la regulación de emociones positivas, cobra relevancia para las personas y las organizaciones humanas el tema de su desarrollo, intervención y aprovechamiento. A continuación se examina la manera de auspiciar su desarrollo en contextos sociales y de trabajo.

\section{Criterios de intervención}

Entre diversos criterios que deben considerarse para realizar con éxito un proceso de intervención, a continuación se examinan algunos, inspirados en evidencias de la psicología positiva. Su aplicación puede facilitar el diseño 
de experiencias educativa para el desarrollo del capital psicológico y de mecanismos de regulación de emociones positivas de personas y grupos en el trabajo.

1. Evaluación realista de la necesidad se refiere a un conjunto de consideraciones que permiten juzgar la conveniencia y la manera de diseñar y llevar a cabo una intervención (Nielsen, 2013; Nielsen, Abildgaard y Daniels, 2014). De acuerdo con estos estudios, gracias a respuestas bien fundamentadas a las siguientes preguntas será posible verificar la necesidad de la acción y la manera de orientarla: (1) Qué puede servir. Esto implica una clara comprensión del problema o situación que se busca atender y la elección de la más apropiada entre varias opciones de tratamiento. Para ese propósito la participación de empleados, supervisores y gerentes es necesaria. (2) Para quién sirve la intervención. Se precisa definir claramente los públicos objetivo a los cuales se dirigirá la acción. El análisis de sus características provee criterios para dar especificidad a la acción. (3) En qué circunstancias será eficaz. Supone tomar en consideración realidades contextuales diversas que podrán facilitar o aún dificultar el proceso. (4) Porqué hacerlo de la manera prevista o propuesta. La respuesta debe considerar antecedentes teóricos, metodológicos y demás resultados de investigación que soporten la eficacia de las acciones previstas.

2. Identificar y tratar preventivamente las posturas y actitudes de las personas en relación con el cambio que se desea promover es otra consideración necesaria para Wright, Van der Heijden, Burt, Bradfield y Cairns (2008). En particular los líderes que intervienen en el proceso tienen un rol clave en la promoción del compromiso y participación de sus equipos de trabajo, en la claridad del objetivo y el significado del cambio.

3. Identificar y desarrollar las fortalezas personales es una recomendación de Le Blanc y Oerlemans (2016), para mejorar la efectividad y las posibilidades de éxito. Significa identificarlas, desarrollarlas y estimular su uso, como estrategia para remover las resistencias, promover la autoestima, autoeficacia y el compromiso con la mejora. Esto no supone ignorar las limitaciones o deficiencias sino apalancar el cambio en las condiciones positivas con que ya cuentan las personas.

4. Salanova, Llorens, Acosta y Torrente (2013) desarrollaron el modelo HERO (Healthy \& Resilient Organizations) que propone estrategias focalizadas en empleados, equipos y organización con el propósito de promover desempeño óptimo, salud, calidad de vida laboral y calidad del desempeño organizacional. Esta aproximación multinivel es necesaria para que el proceso tenga efectos sobre todas las estructuras y las instancias de la organización. Para que los cambios en un nivel no sean neutralizados u obstaculizados por otros el modelo sugiere considerar cinco campos de intervención para incidir sobre los equipos de trabajo y sobre la organización: (1) Valoración y evaluación de los procesos de atracción de candidatos, reclutamiento, selección, retención, monitoreo del contrato psicológico, educación; (2) diseño y rediseño del trabajo para mejorar la provisión de recursos sociales y de tarea, de prácticas organizacionales y del contexto del trabajo; (3) liderazgo transformacional, en el que se educará a los líderes de todos los niveles para desarrollar los atributos de carisma, inspiración, visión y promoción de optimismo, esperanza y resiliencia; (4) entrenamiento para desarrollo y promoción de la auto-eficacia; (5) manejo de la carrera para creación de posibilidades de desarrollo ocupacional y promoción de la empleabilidad. Para intervenir al nivel de los individuos se sugiere en el modelo acudir a estrategias conductuales, cognitivas y volicionales, que suponen la práctica de conductas sociales positivas, la reflexión sobre la conveniencia de tales conductas y la disposición a favorecerlas por la satisfacción que proveen y las respuestas positivas de las personas afectadas, según lo evidencian Lyubomirsky y 
Layous (2013).

5. Un último elemento importante de mencionar en esta lista de criterios de intervención es el clima emocional o clima de afecto. Para Sekerka y Fredrickson (2008) el clima emocional es un ambiente que se constituye y se percibe a partir de las emociones positivas que se experimentan en el trabajo; aporta al significado de la tarea y del rol organizacional, estimula el logro, el compromiso y el significado de las conexiones sociales, contribuye a incrementar la identificación con la organización y al crecimiento y desempeño organizacional. El clima de afecto para Parke y Seo (2017) se refiere a los afectos que promueven las organizaciones entre los empleados por razón de las experiencias y manifestaciones afectivas que permiten y los modos particulares de expresar y manejar las emociones y estados de ánimo deseables e indeseables. Estos investigadores proponen una tipología de climas de afecto que relacionan con diferentes tipos de desempeño. Los dos estudios referidos llaman la atención acerca de que en las organizaciones se crean climas emocionales o afectivos que se constituyen en mediadores entre las prácticas y el desempeño, pero también permiten legitimar y sostener competencias afectivas, tales como las que integran el capital psicológico y otras examinadas por la psicología positiva. Por tanto, es clave intervenir el clima afectivo para crear un ambiente de cultivo de emociones positivas y regular las emociones negativas y sus efectos. Este clima es un elemento estratégico para el desarrollo y conservación de niveles convenientes de capital psicológico.

En resumen, la evaluación realista de necesidades, el tratamiento previo de personas participantes en la intervención, identificar $\mathrm{y}$ gestionar el desarrollo de fortalezas además de tratar las deficiencias, tener en consideración el complejo contexto organizacional en el que se gestionarán los cambios y gestionar un adecuado clima afectivo son previsiones necesarias para aportar a la calidad de un plan de desarrollo del capital psicológico de las personas y los equipos en una organización. El manejo de estas condiciones será facilitador potente del plan de intervención que se expone a continuación.

\section{Plan de intervención}

En este aparte se proponen los elementos básicos de un plan de intervención con las siguientes características: (1) Centrarse exclusivamente en el concepto de capital psicológico y sus cuatro componentes. (2) Proponer un objetivo, contenidos y metodología que deberán especificarse $\mathrm{y}$ adecuarse a las realidades de la situación que se quiera intervenir. (3) Orientarse a desarrollar también habilidades de regulación de emociones que aseguren la conservación de las competencias adquiridas o mejoradas. (4) Asegurar la conservación en el tiempo de lo aprendido. (5) Centrarse en el cambio individual mediante una experiencia colectiva.

Objetivo general. Mejorar el capital psicológico de los participantes, en cada uno de sus cuatro componentes, de modo que el aprendizaje logrado permanezca por un período superior a tres meses.

Resultados Esperados. Como consecuencia de su participación en esta experiencia los asistentes estarán en condición de:

1. Identificar limitaciones y fortalezas de su capital psicológico, incluidos sus componentes de esperanza, auto-eficacia, optimismo y resiliencia.

2. Identificar circunstancias del trabajo actual en las que se afecta de modo positivo y también negativo su capital psicológico.

3. Valorar la conveniencia de mantener en un alto nivel su capital psicológico. 
4. Identificar mecanismos personales de regulación de emociones positivas.

5. Comprender y practicar actividades de regulación de emociones que le permitan conservar y desarrollar su capital intelectual.

6. Proponer acciones de aprovechamiento de circunstancias positivas del trabajo y de cambio de las negativas, para el desarrollo del capital psicológico.

\section{Diseño de la intervención}

\section{Actividades preliminares.}

(a)

Identificar y evaluar con claridad la situación que se espera resolver o corregir con la intervención. (b) Verificar la comprensión y la intención de los distintos interesados: participantes, supervisores, gerentes, clientes o compañeros. (c) Responder las preguntas de la evaluación realista de la situación: qué, para quién, en qué circunstancias, por qué? (d) Acordar con los interesados los objetivos y el plan de trabajo. (e) Varios días antes del inicio hacer primera evaluación del capital psicológico y del desempeño de los participantes y del o los grupos de control con los que se hará el contraste.

2. Contenidos que se presentarán. (a) Selección y presentación de contenidos diversos que se tratarán en el proceso: capital psicológico, esperanza, auto-eficacia, optimismo, resiliencia; mecanismos de regulación de emociones (saboreo y atenuación); efectos cognitivos, conductuales, laborales y personales del capital intelectual y de sus componentes. (b) Elaboración de documentos y presentaciones, búsqueda de películas o videos, conferencias, casos o ejercicios que permiten una presentación clara y atractiva de los contenidos necesarios.

3. Procedimiento. Se presentan a continuación actividades agrupadas por sesiones de manera que se facilite estimar el tiempo y los efectos esperados de cada sesión.
Primera sesión. Incluye actividades para desarrollar los objetivos 1 y 2. Su duración estimada es de cuatro horas. (a) Presentación del facilitador, de los participantes, del plan general de intervención y del plan de trabajo de la sesión. (b) Presentación de los conceptos de capital psicológico, esperanza, auto-eficacia, optimismo y resiliencia. Dar ilustraciones y ejemplos. Pedir a los participantes ejemplos derivados de su experiencia personal. (c) Presentar a cada participante los resultados de la evaluación previa de su capital psicológico. Mostrar los resultados y tendencias colectivas en el grupo de participantes. Analizar con el grupo estas tendencias. (d) Presentar resultados de investigación que evidencian los efectos positivos individuales, grupales y organizacionales de cada uno de los elementos del capital psicológico. Pedir a los participantes ejemplos personales de consecuencias positivas o negativas. (e) Al terminar, ofrecer síntesis de la sesión.

Segunda sesión. Se realiza una semana después de la anterior. Desarrolla los objetivos 3 y 4. Se trabajará con cada uno de los componentes y con el concepto de capital psicológico, por lo que habrá que destinar tiempo para cada uno de los temas por separado. Esta sesión podrá durar entre seis y ocho horas, dependiendo del número de participantes y del interés que se logre despertar en ellos y en el equipo. (a) Individualmente identificar las circunstancias del trabajo actual que dificultan y las que facilitan el desarrollo del capital psicológico personal. (b) Presentar a los compañeros del equipo los resultados de su búsqueda y posibles explicaciones de sus causas. Recibir observaciones y sugerencias de los compañeros. (c) Presentar el concepto de regulación de emociones, con ejemplos y casos ilustrativos. (d) De modo individual elaborar planes para aprovechamiento de las circunstancias favorables y mejora de las desfavorables para cada uno de los componentes del capital psicológico. Especificar: objetivo, acciones, limitaciones posibles, acciones alternas en caso de dificultades 
previstas, estrategias de regulación más apropiadas o necesarias. (e) Presentar a los compañeros de equipo el plan personal elaborado, solicitar comentarios y sugerencias. Hacer los ajustes necesarios. (f) Elaborar documento sencillo con los elementos centrales del plan, plazos, recursos y resultados esperados y presentarlo al facilitador. (g) Evaluar de nuevo el capital intelectual, como se hizo preliminarmente. Cerrar la sesión.

Tercera sesión. Se realiza un mes después de la anterior. Tiene como propósito refrescar los conceptos aprendidos en las dos primeras sesiones y evaluar los logros del plan establecido. (a) Repaso de conceptos y de experiencias obtenidas en las sesiones previas. (b) Presentación por cada participante de logros, fracasos y soluciones dadas. De ser posible, recibir retro-comunicación de compañeros de equipo. (c) Celebrar los logros. (d) Reformular o ajustar el plan en caso de ser necesario. (e) Evaluar colectivamente los avances y las dificultades y elaborar conclusiones colectivas generales acerca de correctivos y posibilidades de continuar el plan.

Esta sesión puede realizarse individualmente por video conferencia, chat o correo electrónico en cuyo caso el interlocutor será el facilitador de la intervención. Puede repetirse uno o dos meses después para consolidar los procesos de memoria y mantener los efectos obtenidos.

Cuarta sesión. Se trata de una reunión de trabajo con quien lideró el proceso por parte de la empresa. El facilitador informará sobre los resultados generales del proceso y dará recomendaciones para el sostenimiento posterior de los efectos conseguidos. Informará sobre la importancia de contar con procedimientos y con un liderazgo y clima que estimulen las conductas aprendidas. Tres y seis meses después se evaluará nuevamente el capital psicológico y el desempeño de los participantes, luego de lo cual se presentará un informe general, escrito, para quien solicitó este servicio.
Este mismo plan puede llevarse a cabo con un equipo de trabajo y trascender del tratamiento individual al grupal. En este caso los participantes examinarán experiencias y realidades del trabajo comunes al equipo y las soluciones propuestas tendrán como referente el equipo de trabajo y no cada participante en particular.

4. Evaluación. Esta evaluación se debe adecuar al nivel individual o grupal en el que se desarrolló la intervención. Puede tener dos propósitos distintos. Uno será verificar la calidad y conveniencia de todo el plan de intervención. Para el propósito se examinará la capacidad de los distintos elementos para producir el cambio esperado: objetivos, contenidos, procedimientos, duración, facilitador, incidencia de variables contextuales, participación y rol de distintos agentes, circunstancias especiales. De este modo se podrá juzgar la validez de la estrategia. Esto será de particular utilidad si se trata de una experiencia de intervención que deberá realizarse en diversas ocasiones y con grupos distintos (Nielsen, 2013; Nielsen et al., 2014). De este modo se asegura su validez.

Otro enfoque, complementario del precedente, pero que puede realizarse con independencia de él, es la evaluación de la eficacia de la estrategia. Tiene como propósito examinar la capacidad de todo el conjunto para producir los cambios previstos en los objetivos y estimar la permanencia de tales cambios. Esta evaluación es más conveniente cuando la acción se desarrolle en grupos conformados por personas independientes o cuando solo se realice esporádicamente. Será preciso contar con instrumentos psicométricos que evalúen cada una de las variables objeto de tratamiento. Deseable contar con formas paralelas de modo que se disminuya el error asociado a la administración repetida del mismo instrumento. Será preciso también definir varios indicadores de desempeño que permitan registrar y medir actuaciones laborales que reflejen la presencia de los constructos examinados y que puedan ser registrados por supervisores, clientes, 
compañeros de trabajo u otros observadores. Estos indicadores se correlacionarán con los resultados de los instrumentos psicométricos y se harán comparaciones entre los distintos tiempos en los que se registraron las medidas. Será de utilidad realizar comparaciones por género, edad, especialidad o tipo de trabajo para examinar cómo estas condiciones pueden tener un papel moderador en la obtención de los resultados. Es igualmente conveniente, en la medida de lo posible, examinar la participación de condiciones mediadoras como algunas características de la cultura, el clima organizacional o las prácticas de gestión humana.

\section{Conclusión}

El plan de intervención descrito propone un acercamiento metódico al desarrollo del capital psicológico de personas y de equipos de trabajo. Para mejorar la duración de los aprendizajes en el tiempo, lo que no han previsto otras estrategias de intervención referidas en este escrito, se incorporan varios criterios: prolongación en el tiempo de la experiencia de aprendizaje, desarrollo de habilidades de regulación positiva de emociones y una estrategia de aprendizaje participativo, basado en experiencias personales $\mathrm{y}$ en retro comunicación del facilitador y de otros participantes. Este plan de trabajo está pensado inicialmente para ser una experiencia de aprendizaje, en grupo, de personas que no conforman un equipo de trabajo. No obstante, su estructura permite convertir el proceso en una experiencia de equipo de trabajo. Será preciso ponerla en práctica para poderla evaluar, pero sus fundamentos en el saber actual teórico y empírico da garantía de validez de contenido. Naturalmente que existen otras posibilidades de elaborar la intervención, que podrán adoptarse en la medida en que los resultados de la evaluación sugieran mejoras o correctivos posibles.

\section{Referencias}

Avey, J. B., Reichard, R. J., Luthans, F. \& Mhatre, K. H. (2011). Meta-Analysis of the Impact of Positive Psychological Capital on Employee Attitudes, Behaviors, and Performance. Human Resource Development Quarterly, 22(2), 127-152, DOI: $10.1002 / \mathrm{hrdq} .20070$.

Eichenbaum H. B. (2003). Learning and memory: Brain systems. In: Squire L. R, Bloom F. E, McConnell S. K, Roberts J. L, Spitzer N. C, Zigmond M. J, (Editors). Fundamental Neuroscience. Academic Press: New York, NY, 1299-1328.

Ernst, M., Nelson, E. E., Jazbec, S., McClure,E. B., Monk, C.S., Leibenluft, E. Blair, J. \& Pine, D.S. (2005). Amygdala and nucleus accumbens in responses to receipt and omission of gains in adults and adolescents. NeuroImage, 25, 1279-1291. DOI:10.1016/j.neuroimage.2004.12.038

Garnefski, N., Teerds, J., Kraaij, V., Legerstee, J., \& van den Kommer, T. (2004). Cognitive emotion regulation strategies and depressive symptoms: Differences between males and females. Personality and Individual Differences, 36(2), 267-276. DOI:10.1016/ S0191-8869(03)00083-7

Gross, J. J., Richards, J. M., \& John, O. P. (2006). Emotion regulation in everyday life. In D. K. Snyder, J. A. Simpson, \& J. N. Hughes (Eds.), Emotion regulation in families: Pathways to dysfunction and health (pp. 13-35). Washington, DC: American Psychological Association

Hobfoll S. (2002). Social and psychological resources and adaptation. Review of General Psychology, 6(4), 307 - 324.

Knutson, B., Wimmer, G. E., Kuhnen, C. \& Winkielman, P. (2008). Nucleus accumbens 
activation mediates the influence of reward cues on financial risk-taking. MPRA Paper No. 8013, 1-18. Recuperado de http://mpra. ub.uni-muenchen.de/8013/

Kelly, J. R. \& Barsade, S. G. (2001). Mood and Emotions in Small Groups and Work Teams. Organizational Behavior and Human Decision Processesit'pol. 86(1), 9-130

Le Blanc, P. M. \& Oerlemans, W. G. M. (2016). Amplition in the work place: building a sustainable workforce through individual positive psychological interventions. Papeles del Psicólogo, 37(3), 185-191.

Lopes, P.N., Mezlek, J. B., Extremera, N., Hertel, J., Fernández, P., Schultz, A., \& Salovey, P. (2011). Emotion Regulation and the Quality of Social Interaction: Does the Ability to Evaluate Emotional Situations and Identify Effective Responses Matter? Journal of Personality, 79(2), 429-467, DOI:10.1111/j.1467-6494.2010.00689.x

Lyubomirsky, S. \& Layous, K. (2013). How do Simple Positive Activities Increase Well-being? Current Directions in Psychological Science, 22(1), 57-62, DOI: 10.1177/0963721412469809

Luthans F., Youssef, C. M., Avolio, B. J. (2007a). Psychological capital. New York: Oxford University Press.

Luthans, F, Avolio, B. J., Avey, J. B. \& Norman, S. M. (2007b). Positive Psychological Capital: Measurement and Relationship with Performance and Satisfaction. Personnel Psychology 60, 541-572

Luthans, F., Avey, J. B. \& Patera, J. L. (2008). Experimental Analysis of a Web-Based. Training Intervention to Develop Positive Psychological Capital. Academy of Management Learning \& Education, 7 (2), 209-221
Luthans, F., Avey, J. B., Avolio, B. J. \& Peterson, S. J. (2010). The Development and Resulting Performance Impact of Positive Psychological Capital. Human Resource Development Quarterly, 21(1), 41 - 67 . DOI: 10.1002/hrdq.20034

Luthans, B.C., Luthans, K. W., Avey, J. B. (2013). Building the leaders of tomorrow: The development of academic psychological capital. Journal of Leadership and organizational Studies, 21(2), 191 - 199, DOI: $101177 / 1548051813517003$

Nielsen, K. (2013). Review Article: How can we make organizational interventions work? Employees and line managers as actively crafting interventions. Human Relations, 66(8), 1029-1050. DOI: $10.1177 / 0018726713477164$

Nielsen, K., Abildgaard, J. S. \& Daniels, K. (2014). Putting context into organizational intervention design: Using tailored questionnaires to measure initiatives for worker well-being. Human Relations, $67(12)$

Parke, M. R. \& Seo, M-G. (2017). The role of affect climate in organizational effectiveness. Academy of Management Review, 42 (2), 334-360. DOI:10.5465/amr.2014.0424

Peterson, S.J., Luthans, F., Avolio, B. J., Walumbwa, F. O. \& Zhang, Z. (2011). Psychological Capital and Employee Performance: A Latent Growth Modeling Approach. Personnel Psychology 64(2), 427-450. DOI: 10.1111/j.17446570.2011.01215.x

Quoidbach, J., Berry, E. V., Hansenne, M. \& Mikolajczak, M. (2010). Positive emotion regulation and well-being: Comparing the impact of eight savoring and dampening strategies. Personality and Individual Differences, DOI:10.1016/j. paid.2010.03.048 
Salimpoor, V. N., Bosch, I., Kovacevic, N., Mcintosh, A. R., Dagher, A. \& Zatorre, R. J. (2013). Interactions Between the Nucleus Accumbens and Auditory Cortices Predict Music Reward Value. Science, 340, 216219 DOI: $10.1126 /$ science. 1231059

Sekerka, L. E. \& Fredrickson, B. L. (2008). Establishing positive emotional climates to advance organizational transformation. In N. A. Ashkanasy \& C. L. Cooper. (Eds), Research Companion to Emotion in Organizations (pp. 531-545), Northampton, MA: Edward Elgar Publishing.

Silvers, J. \& Ochsner, K. N. (2016). Toward a personalized science of emotional regulation. Social and Personality Psychology Compass, 10(4), 171-187. DOI: $10.1111 / \mathrm{spc} 3.12240$

Squire, L. R. (2004). Memory systems of the brain: A brief history and current perspective. Neurobiology of Learning and Memory, 82, 171-177

Russo, S., D \& Stoycova, P. (2015). Psychological Capital Intervention (PCI): A Replication and Extension. Human Resource Development Quarterly, 26(3), 329 - 347, DOI: $10.1002 /$ hrdq. 21212

Salanova, M., Llorens , S., Acosta, H. \& Torrente, P. (2013). Positive Interventions in Positive Organizations. Terapia Psicológica, 31(1), 101-113

Snyder, C. R. 1994. Hope and optimism. Encyclopedia of Human Behavior. 535542. San Diego, CA: Academic Press.

Tsai, W-C.(2001). Determinants and consequences of employee displayed positive emotions. Journal of Management, 27, 497-512
Trejo, B. C., Richard, E.M., van Driel, M. \& McDonald, D.P. (2015). Cross cultural competence: The role of emotion regulation ability and optimism. Military Psychology, 27(5), 276 - 286. DOI: 101037/mil0000081

Tugade M. M \& Fredrickson B. L. (2007). Regulation of Positive Emotions: Emotion Regulation Strategies that Promote Resilience. Journal of Happiness Studies, 8, 311-333. DOI: 10.1007/s10902-0069015-4

Warr, P., Bindull, U. K., Parker, S. K. \& Inceoglu, I. (2013). Four-Quadrant Investigation of Job-related Affects and Behaviours. Journal of Work and Organizational Psychology. DOI: 10.1080/1359432X.2012.744449

Wegener, D.T. \&. Petty, E. E. (1994), Moodmanagement across affective states: The hedonic contingency hypothesis. Journal of Personality and Social Psychology 66, pp. 1034--1048.

Wingerden, J., Bakker, A. B. \& Derks, D. (2016). A test of a job demands-resources intervention. Journal of Managerial Psychology, 31(3), 686 - 701. DOI: 10.1108/JMP-03-2014-0086

Wright, G., Van der Heijden, K., Burt, G., Bradfield, R. \& Cairns, G. (2008) 'Scenario planning interventions in organizations: an analysis of the causes of success and failure.', Futures, 40 (3), 218-236.

Yoo, J., Miyamoto, Y. \& and Ryff, C. D. (2016). Positive Affect, Social Connectedness, and Healthy Biomarkers in Japan and the U.S. Emotion, 16(8), 1137-1146 